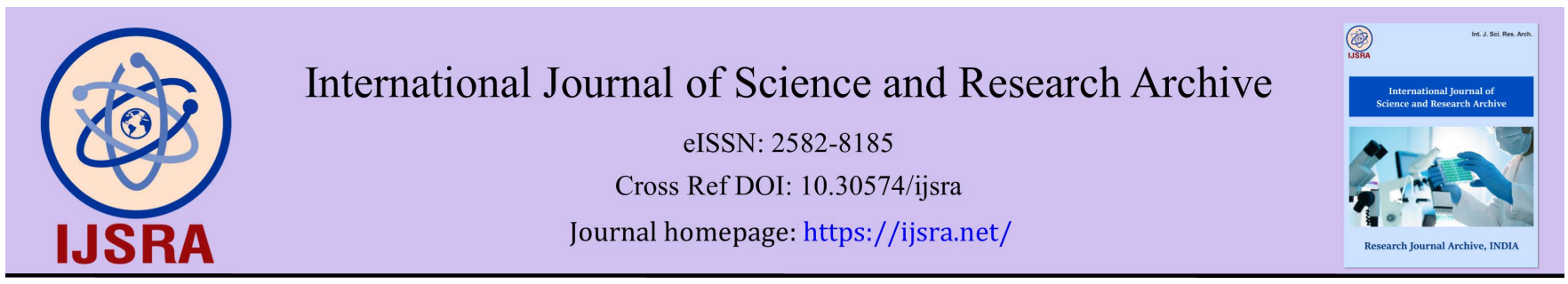

(RESEARCH ARTICLE)

Check for updates

\title{
Assessing the incidence of acute kidney injury with combination vancomycin and piperacillin-tazobactam therapy compared to that of vancomycin and cefepime
}

\author{
Sydney Jean Rechtenbaugh ${ }^{1}$, Fekadu Fullas ${ }^{1,}{ }^{*}$, Kimberly Ann Zellmer ${ }^{1}$, Joseph Michael Buren ${ }^{2}$, Corey James \\ Thieman ${ }^{1}$, and Michael Theodore Padomek ${ }^{1}$
}

1 UnityPoint Health-St. Luke's, Pharmacy Department, 2720 Stone Park, Boulevard, Sioux City, IA 51104, USA.

2 Sanford USD Medical Center, Pharmacy Department,1305 W 18 th St., Sioux Falls, SD 57117-5039; USA.

International Journal of Science and Research Archive, 2021, 02(01), 143-148

Publication history: Received on 03 February 2021; revised on 09 March 2021; accepted on 11 March 2021

Article DOI: https://doi.org/10.30574/ijsra.2021.2.1.0040

\begin{abstract}
Previous studies have shown a correlation between acute kidney injury (AKI) and combination antimicrobial therapy comprising piperacillin-tazobactam and vancomycin. In this study, AKIs were compared in patients who received vancomycin plus piperacillin-tazobactam with those who received vancomycin plus cefepime. We found a statistically significant increase in AKI risk in the vancomycin plus piperacillin-tazobactam group when compared to the vancomycin plus cefepime group using both AKI criteria [KDIGO $18.9 \%$ vs. $4.5 \%$ ( $p=0.0012$ ); RIFLE $11.2 \%$ vs. $1.8 \%$ ( $p$ $=0.0029)]$. Vancomycin in combination with piperacillin-tazobactam led to an increased risk of AKI in comparison to vancomycin and cefepime.
\end{abstract}

Keywords: Vancomycin Plus Piperacillin-Tazobactam; Vancomycin Plus Cefepime; AKI; KDIGO; RIFLE; Nephrotoxicity; Antibiotics, Antimicrobials

\section{Introduction}

Acute kidney injury (AKI) has been linked to increased morbidity and mortality [1]. Drug induced AKI is a common adverse reaction in hospitals, with such episodes being frequent in hospitalized patients who receive antimicrobial agents [2]. Proper management of these agents reduces the incidence of AKI [3]. Analysis of over 20,000 antimicrobialrelated AKI events by the United Sates FDA ranked vancomycin and penicillin combinations as the third and fifth most common causes of AKI, respectively [4]. Antimicrobial therapy with vancomycin is known to increase the risk of AKI and nephrotoxicity. To avoid vancomycin-induced nephrotoxicity, practitioners target trough concentrations, monitor drug levels, and avoid concurrent nephrotoxic agents when possible [5]. Vancomycin is frequently used in combination with other broad-spectrum antimicrobials. Piperacillin-tazobactam and cefepime are commonly added to vancomycin for empiric antipseudomonal coverage in healthcare-associated infections. Recent studies have shown an increased risk of AKI when vancomycin is combined with piperacillin-tazobactam in comparison to other agents [6,7]. In a study by Navalkale et al, it was shown that the incidence of AKI was higher with the combination of vancomycin-piperacillintazobactam therapy when compared with vancomycin-cefepime therapy [8]. In another study involving 718 patients, the combination of vancomycin and piperacillin-tazobactam was shown to be more nephrotoxic than vancomycin alone [9]. In a much larger study of 11,650 patients by Rutter et al [10], a combination therapy of vancomycin and piperacillintazobactam resulted in a significantly higher incidence of AKI than monotherapy with either agent separately.

The current retrospective analysis of patients treated with combination of vancomycin and piperacillin-tazobactam in comparison to those treated with vancomycin and cefepime was completed to educate providers on the incidence of $\mathrm{AKI}$ and assist pharmacists in developing safe and effective antimicrobial therapy recommendations.

\footnotetext{
* Corresponding author: Fekadu Fullas

UnityPoint Health-St. Luke's, Pharmacy Department, 2720 Stone Park, Boulevard, Sioux City, IA 51104, USA.
}

Copyright $@ 2021$ Author(s) retain the copyright of this article. This article is published under the terms of the Creative Commons Attribution Liscense 4.0 


\section{Methods}

The study was approved by the Siouxland Institutional Review Board. It was a single-center, retrospective cohort study, completed at a 154-bed community hospital in Sioux City, Iowa. The incidence of AKI was compared between two groups of patients receiving either combination vancomycin and piperacillin-tazobactam or vancomycin and cefepime. The primary objective was to determine the rate of AKI with inpatient antimicrobial therapy of piperacillin-tazobactam plus vancomycin compared to cefepime plus vancomycin. AKI was defined by using Kidney Disease Improving Global Outcomes (KDIGO) and Risk, Injury, Failure, Loss of kidney function, and End-stage kidney disease (RIFLE) criteria. AKI by KDIGO definition is stratified by stages with stage 1 defined as an increase in serum creatinine (SCr) $\geq 0.3 \mathrm{mg} / \mathrm{dL}$ within 48 hours or an increase 1.5-1.9 times baseline within the prior 7 days, stage 2 is an increase in SCr 2.0-2.9 times baseline, and stage 3 is an increase in SCr 3.0 times baseline or to $\geq 4.0 \mathrm{mg} / \mathrm{dL}$, or initiation of renal replacement therapy [11]. RIFLE criteria define AKI as an increase in SCr 1.5 times baseline (Risk), an increase in SCr 2 times baseline (Injury), or an increase in SCr 3 times baseline (Failure) [12]. The secondary objective was to evaluate the average length therapy, length of hospital stay and comorbidities between the two groups. The respective Charlson Co-morbidity Index [13] for the two groups was computed for co-morbidity parameters. All vancomycin, piperacillin-tazobactam, and cefepime orders from January 2019 to October 2019 were reviewed and assessed for combination therapy. Those who received combination therapy for 48 hours or longer were reviewed, and the following information was gathered: age, gender, diagnoses, comorbidities, serum creatinine levels, vancomycin trough levels, length of stay, and concurrent antiinfective/nephrotoxic agents. The hypothesis of this study is combination piperacillin-tazobactam plus vancomycin will lead to an increased incidence of acute kidney injury in comparison to vancomycin and cefepime.

Patients' charts were retrospectively reviewed for inclusion and exclusion criteria. Patients aged 18 years and older were included in the study if they were treated with combination vancomycin and piperacillin-tazobactam therapy or vancomycin and cefepime therapy for at least 48 hours. Patients also must have had a baseline SCr level at least within 48 hours prior to the initiation of antimicrobial therapy. Exclusion criteria included patients without a baseline SCr level at least 48 hours prior to antimicrobial therapy initiation, or absence of at least one follow-up SCr. Other exclusions included baseline SCr levels greater than $4.0 \mathrm{mg} / \mathrm{dL}$, diagnosis of end-stage renal disease, AKI with need for continuous renal replacement therapy at antibiotic initiation, or if the patient expired during the hospital admission.

Statistical analyses were performed by using either the chi-square tests $\left(\chi^{2}\right)$, or a two-tailed Fisher Exact Test. A twotailed Student t-test was employed to compare age, SCr levels, total days of therapy and length of stay between the two groups. The $\chi^{2}$ test was run by using the Yate's correction to prevent overestimation of statistical significance. The twotailed Fisher Exact Test was employed for all data set where individual values were less than 5 (Tables 1 and 2 ).

\section{Results}

In the piperacillin-tazobactam/vancomycin group, a total of 363 patients were evaluated for inclusion and exclusion criteria. Of these patients, 163 were excluded because of discontinuation of the combination therapy within 48 hours after initiation. A further 44 patients expired within the review period, and 13 patients had a diagnosis of end-stage renal disease and/or had a baseline SCr level of greater that $4 \mathrm{mg} / \mathrm{dL}$. In the cefepime/vancomycin arm, a total of 317 patients were assessed for inclusion and exclusion criteria. Of these, 159 patients received the combination therapy for less than 48 hours, 30 patients expired and 17 had end-stage renal disease and/or had SCr level greater than $4 \mathrm{mg} / \mathrm{dL}$ on initiation of therapy. After identifying exclusions, 143 patients in the piperacillin-tazobactam plus vancomycin group and 111 patients in the cefepime plus vancomycin group were included in the study. The average age of patients in the piperacillin-tazobactam and cefepime group was 59.09 and 64.81 years, respectively. The majority were male patients with $94(65.7 \%)$ in the piperacillin-tazobactam-vancomycin group and 62 (55.9\%) in the cefepime-vancomycin group. Most baseline characteristics, such as gender distribution, SCr values and total days of therapy were similar between groups (Table 1). There were no significant differences in nephrotoxic agents received, total vancomycin doses, or vancomycin trough levels between groups.

Table 1 Characteristics of the Cefepime-Vancomycin and Piperacillin-Tazobactam-Vancomycin Groups of Patients

\begin{tabular}{|l|l|l|l|}
\hline & $\begin{array}{l}\text { Cefepime-Vancomycin } \\
(n=111)\end{array}$ & $\begin{array}{l}\text { Piperacillin-tazobactam- } \\
\text { Vancomycin }(n=143)\end{array}$ & $p$ \\
\hline Characteristic*
\end{tabular}




\begin{tabular}{|c|c|c|c|}
\hline Gender (male/female) & $62 / 49$ & $94 / 49$ & 0.1407 \\
\hline Age (Mean \pm S.D. $[\mathrm{yrs}])$ & $64.801 \pm 16.586$ & $59.098 \pm 17.665$ & 0.0092 \\
\hline \multicolumn{4}{|l|}{$\mathrm{SCr}(\mathrm{mg} / \mathrm{dL})$} \\
\hline \multicolumn{4}{|l|}{ Mean \pm S.D. } \\
\hline (Baseline) & $1.0006 \pm 0.4325$ & $1.0395 \pm 0.4625$ & 0.5538 \\
\hline (After treatment) & $0.88426 \pm 0.3552$ & $1.0430 \pm 0.4846$ & 0.0041 \\
\hline \multicolumn{4}{|l|}{ Total days of therapy } \\
\hline Mean \pm S.D. & $4.59 \pm 2.98$ & $4.32 \pm 2.39$ & 0.4193 \\
\hline \multicolumn{4}{|l|}{ LOS (days) } \\
\hline Mean \pm S.D. & $6.9189 \pm 6.9871$ & $7.3287 \pm 5.1070$ & 0.5898 \\
\hline Radio-contrast dyes & 53 & 64 & 0.8991 \\
\hline \multicolumn{4}{|l|}{ Diagnosis } \\
\hline \multicolumn{4}{|c|}{ PNA/Respiratory failure/ } \\
\hline Lung infection & 60 & 53 & 0.0100 \\
\hline SSTI & 10 & 35 & 0.0024 \\
\hline Sepsis & 16 & 22 & 0.8611 \\
\hline Abdominal infection & 3 & 9 & 0.2987 \\
\hline Osteomyelitis & 1 & 8 & 0.0819 \\
\hline Bacteremia & 1 & 2 & 1 \\
\hline Cystitis & 3 & 2 & 0.6559 \\
\hline Neutropenic fever & 5 & 1 & 0.0889 \\
\hline Other infections & 12 & 11 & 0.5219 \\
\hline
\end{tabular}

*S.D. = standard deviation; $\mathrm{yrs}=$ years; $\mathrm{SCr}=$ serum creatinine; $\mathrm{LOS}=$ length of stay; PNA = pneumonia; SSTI = skin and soft tissue infection.

Table 2 Comparison of AKI with vancomycin plus cefepime versus vancomycin plus piperacillin-tazobactam

\begin{tabular}{|l|l|l|l|}
\hline $\begin{array}{l}\text { Kidney Function } \\
\text { Evaluation }\end{array}$ & $\begin{array}{l}\text { Vancomycin and } \\
\text { Cefepime (n=111) }\end{array}$ & $\begin{array}{l}\text { Vancomycin and } \\
\text { piperacillin-tazobactam } \\
\text { (n=143) }\end{array}$ & $\boldsymbol{p}^{*}$ \\
\hline KDIGO Criteria & $18(12.6 \%)$ & \\
\hline Stage 1 AKI event rate & $4(3.6 \%)$ & $8(5.6 \%)$ & 0.0128 \\
\hline Stage 2 AKI event rate & $1(0.9 \%)$ & $1(0.7 \%)$ & 0.0819 \\
\hline Stage 3 AKI event rate & 0 & $27(18.9 \%)$ & 1 \\
\hline Total AKI & $5(4.5 \%)$ & & 0.0012 \\
\hline RIFLE Criteria & \multicolumn{5}{|l|}{} & \\
\hline Risk & $1(0.9 \%)$ & $8(5.6 \%)$ & 0.0819 \\
\hline Injury & $1(0.9 \%)$ & $8(5.6 \%)$ & 0.0819 \\
\hline Failure & 0 & $1(0.7 \%)$ & 1 \\
\hline Total AKI & $2(1.8 \%)$ & $17(11.9 \%)$ & 0.0029 \\
\hline
\end{tabular}

When analyzing patients using the KDIGO criteria, the piperacillin-tazobactam plus vancomycin group had 27 AKI events (18.9\%), and the cefepime plus vancomycin group had 5 events (4.5\%; $p=0.0012$ ) [Table 2]. Event rates using RIFLE criteria were $17(11.9 \%)$ and $2(1.8 \% ; p=0.0029)$ in the piperacillin-tazobactam plus vancomycin and cefepime plus vancomycin groups, respectively [Table 2]. The mean length of hospital stay was 7.3287 days in the piperacillintazobactam plus vancomycin group and 6.9189 days in the cefepime plus vancomycin group ( $p=0.5898$ ) [Table 1]. The pattern of co-morbidities between the two groups is shown in Table 3. 
Table 3 Comparison of co-morbidities between the two groups

\begin{tabular}{|c|c|c|c|}
\hline & \multicolumn{3}{|c|}{ Group of Patients } \\
\hline & $\begin{array}{l}\text { Cefepime-Vancomycin } \\
(n=111)\end{array}$ & $\begin{array}{l}\text { Piperacillin-tazobactam- } \\
\text { Vancomycin }(n=143)\end{array}$ & $p$ \\
\hline \multicolumn{4}{|l|}{ Co-morbidity* } \\
\hline Myocardial infarction & 1 & 4 & 0.3900 \\
\hline Congestive heart failure & 16 & 30 & 0.2367 \\
\hline Peripheral vascular disease & 20 & 23 & 0.8065 \\
\hline \multicolumn{4}{|l|}{ Cardiovascular accident/ } \\
\hline Transient ischemic attack & 9 & 5 & 0.1871 \\
\hline Dementia & 7 & 2 & 0.0443 \\
\hline COPD & 44 & 35 & 0.0128 \\
\hline \multicolumn{4}{|l|}{ Connective tissue } \\
\hline Disease & 0 & 0 & 1 \\
\hline Peptic ulcer disease & 1 & 1 & 1 \\
\hline Liver disease & 8 & 8 & 0.6467 \\
\hline Diabetes mellitus & 29 & 49 & 0.2088 \\
\hline Hemiplegia & 1 & 0 & 1 \\
\hline Moderate to severe CKD & 0 & 0 & 1 \\
\hline Solid tumor & 18 & 17 & 0.4201 \\
\hline Leukemia & 5 & 3 & 0.2810 \\
\hline Lymphoma & 2 & 2 & 1 \\
\hline AIDS & 0 & 0 & 1 \\
\hline \multicolumn{4}{|l|}{ Charlson Co-Morbidity } \\
\hline Index (Mean \pm S.D. $)$ & $4.3783 \pm 2.2965$ & $3.48253 \pm 2.7163$ & 0.0057 \\
\hline
\end{tabular}

${ }^{*} \mathrm{COPD}=$ Chronic Obstructive Pulmonary Disease; $\mathrm{CKD}=$ chronic kidney disease; AIDS = acquired immune deficiency syndrome; S.D. = standard deviation

\section{Discussion}

The most common primary diagnosis for initiation of combinations antimicrobial therapy was pneumonia-respiratory failure-pulmonary infection, with this diagnosis being significantly more frequent $(60 ; 54.1 \%)$ in the cefepimevancomycin group than in the vancomycin-piperacillin-vancomycin group $(53 ; 37.1 \%)[p=0.0100]$. The frequency of skin and soft tissue infections (SSTI) was significantly higher in the latter than the former group (35; $24.5 \%$ versus 10 ; $9.0 \%, p=0.0024)$. In the case of other infections, although differences in frequencies were noted, they were not significant (Table 1).

In this study, combination therapy with piperacillin-tazobactam plus vancomycin led to a statistically significant increase in the incidence of AKI when compared to cefepime plus vancomycin therapy in hospitalized patients. This incidence was observed regardless of AKI evaluation criteria used. Our finding was in line with the results reported by Navalkale et al [8]. SCr levels significantly dropped in the vancomycin-piperacillin-vancomycin group compared with the cefepime-vancomycin group $(p=0.004)$. The mean age of the former group of patients was statistically lower than the latter group ( $\mathrm{p}=0.0092)$ [Table 1]. Concurrent administration of nephrotoxic agents was also evaluated. Both groups had received radio-contrast dyes, with 51 (45.9\%) patients in the cefepime-vancomycin group versus 64 $(32.1 \%)$ in the piperacillin-tazobactam-vancomycin group. However, the difference was not statistically significant $(p=$ 0.8991). Length of hospital stay was slightly shorter in the cefepime plus vancomycin group, but it was not statistically significant $(p=0.5898)$. Previous studies have shown that AKI does lead to increased hospital stay and worsened patient outcomes. Length of hospital stay is frequently used to assess the efficiency of hospital management. Increase in length of hospital stay has been associated with increased risk of opportunistic infections, increased incidence of medication 
side effects and higher mortality rates. Studies have found that shorter hospital stays reduce medical fees which in turn leads to an increase in profit margins and a decrease in overall medical costs [14].

As a secondary objective, this study also analyzed co-morbidities in both groups. Differences, although not statistically significant, were noted in many of the parameters reviewed. Of special note are two exceptions: dementia and chronic obstructive pulmonary disease (COPD), where the difference in frequency of these co-morbidities between the two patient groups were significant, with $p=0.0443$ and 0.0128 for dementia and COPD, respectively. Both COPD (39.6 versus $24.5 \%$ ) and dementia (6.3\% vs $1.4 \%$ ) were more frequent in the cefepime-vancomycin group compared to piperacillin-tazobactam group. However, the overall Charlson Co-Morbidity Index was significantly higher in the piperacillin-tazobactam group $(p=0.0057)$ [Table 3]. Comorbidity burdens, such as diabetes mellitus, coronary artery disease, congestive heart failure, peripheral vascular disease, liver disease, cancer, dementia, chronic kidney disease and acquired immune deficiency syndrome have been reported to be contributory risk factors for AKI [15,16]. Exacerbated COPD is also a major risk factor for AKI [17].

One limitation of this study is that data analysis from a single-center and reviewing patients' charts retrospectively may introduce investigator bias. Another limitation is that the data analyzed were not adjusted for confounding variables, but a comparator group was used to theoretically limit confounding.

\section{Conclusion}

A combination of vancomycin and piperacillin-tazobactam therapy resulted in a significantly higher frequency of AKI when compared to vancomycin and cefepime combination. Based on the results of this study, it may be appropriate to consider limiting the use of combination piperacillin-tazobactam and vancomycin unless other options are not available, due to resistance or/and pathogen-directed coverage. When these agents are used together, pharmacists and providers should be vigilant about the risk of AKI and closely monitor renal function. Education of healthcare providers on the risk of AKI may also help mitigate nephrotoxic antimicrobial combination therapy and guide towards alternative therapies.

\section{Compliance with ethical standards}

\section{Disclosure of conflict of interest}

The authors declare no conflict of interest.

\section{Author contributions}

Sydney Rechtenbaugh, Kimberly Zellmer, Fekadu Fullas and Joseph Buren jointly conceived the idea of the project. Corey Thieman, Michael Padomek and Sydney Rechtenbaugh collected the data. Fekadu Fullas and Sydney Rechtenbaugh analyzed the data and wrote the paper.

\section{References}

[1] Chertow, GM, Burdick E, Honour M, Bonvetre JV, Bates DW. Acute kidney injury, mortality, length of stay, and costs in hospitalized patients. J. Am. Soc. Nephrol. 2005; 16:3365-3370.

[2] Khalili H, Bairami S, Kargar M. Antibiotics induced kidney injury, risk factors, onset time and outcome. Acta. Med. Iran. 2013; 51 (12):817-878.

[3] Mizokami F, Mizuno T. Acute Kidney Injury Induced by Antimicrobial Agents in the Elderly: Awareness and Mitigation Strategies. Drugs Aging. 2015; 32:1-12.

[4] Patek TM, Teng C, Kennedy KE, Alvarez CA, Frei CR. Comparing Acute Kidney Injury Reports Among Antibiotics: A Pharmacovigilance Study of the FDA Adverse Event Reporting System (FAERS). Drug Saf. 2020; 43:17-22.

[5] Luther MK, Timbrook TT, Caffrey AR, Dosa D, Lodise TP, LaPlante KL. Vancomycin plus piperacillin-tazobactam and acute kidney injury in adults: a systematic review and meta-analysis. Crit. Care Med. 2018; 46 (1):2-20.

[6] Yabes J Jr, Stewart L, Shaikh F, Lu DZ, Merritt T, Mende K, Carson L, Robben PM, Leimbach R, Petfield JL, Ganesan A, Tribble DR, Blyth DM. 1929. Risk of Acute Kidney Injury in Combat-Injured Patients Associated with Concomitant Vancomycin and Extended-Spectrum ß-Lactam Antibiotic Use. Open Forum Infect. Dis. 2018 Nov (Suppl 1): S55-556. 
[7] Robertson A, Li C, Hammond DA, Dickey TA. Incidence of acute kidney injury among patients receiving the combination of vancomycin with piperacillin-tazobactam or meropenem. Pharmacotherapy: J Human Pharmacol Drug Ther. 2018; 38 (12):1184-1193.

[8] Navalkele B, Pogue JM, Karino S, Nishan B, Salim M, Solanki S, Pervaiz A, Tashtoush N, Shaikh H, Koppula S, Koon J, Hussain T, Perry W, Evans R, Martin ET, Mynatt RP, Murray KP, Rybak MJ, Kaye KS. Risk of acute kidney injury in patients on concomitant vancomycin and piperacillin-tazobactam compared to those on vancomycin and cefepime. Clin. Infect. Dis. 2017; 64(2):116-123.

[9] Hundeshagen G, Herndon DN, Capek KD, Branski LK, Voigt CD, Killion EA, Cambiaso-Daniel J, Sljivich M, Crescenzo AD, Mlcak RP, Kinsky MP, Finnerty CC, Norbury WB. Co-administration of vancomycin and piperacillintazobactam is associated with increased renal dysfunction in adult and pediatric patients. Crit. Care 2017; 21 (318):1-10.

[10] Rutter WC, Burgess DR, Talbert JC, Burgess DS. Acute kidney injury in patients treated with vancomycin and piperacillin-tazobactam: A retrospective cohort analysis. J. Hosp. Med. 2017; 12 (2):77-82.

[11] Kellem JA, Lameire N, Aspelin P, Barsoum RS, Burdmann EA, Goldstein SL, Herzog CA, Joannidis M, Kribben A, Levey AS, MacLeod AM, Mehta RL, Murray PT, Naicker S, Opal SM, Schaefer F, Schetz M, Uchino S. Kidney Disease: Improving Global Outcomes (KDIGO) Acute Kidney Injury Work Group: KDIGO clinical practice guideline for acute kidney injury. Kidney Int. 2012; 2:1-138.

[12] Bellomo R, Ronco C, Kellum JA, Mehta RL, Palevsky P. Acute renal failure - definition, outcome measures, animal models, fluid therapy and information technology needs: The Second International Consensus Conference of the Acute Dialysis Quality Initiative (ADQI) Group. Crit. Care 2004; 8:R204-R212.

[13] Charlson Comorbidity Index (CCI) - MDCalc. https://www.mdcalc.com/charlson-comorbidity-index-cci (accessed 10 October 2020).

[14] Baek H, Cho M, Kim S, Hwang H, Song M, Yoo S. Analysis of length of hospital stay using electronic health records: A statistical and data mining approach. PLoS One. 2018; 13 (4).

[15] Siew ED, Parr SK, A-Kader K, Eden SK, Peterson JF, Bansal N, Hung AM, Fly J, Speroff T, Ikizler TA, Mathney ME. Predictors of Recurrent AKI. J. Am. Soc. Nephrol. 2016; 27:1190-1200.

[16] Farooqi S, Dickhout JG. Major co-morbid disease processes associated with increased incidence of acute kidney injury. World J. Nephrol. 2016; 5 (2):139-146.

[17] Wan X, Chen D, Tan Y, Ma M, Zhung F, Liu Z, Chen Y, Shao W, Cao C. Incidence, Risk Factors and Prognostic Implications of Acute Kidney Injury in Patients with Exacerbation of COPD. Int. J. Chron. Obstruct. Pulmon. Dis. 2020; 15:1085-1092. 